\title{
LncRNA H19 promotes glioma angiogenesis through miR-138/HIF-1a/VEGF axis
}

\author{
Z. Z. LIU ${ }^{1}$, Y. F. TIAN ${ }^{2}$, H. WU ${ }^{3}$, S. Y. OUYANG ${ }^{4}$, W. L. KUANG ${ }^{1, *}$
}

${ }^{1}$ Department of Oncology, Xiangya Hospital, Central South University, Changsha, China; ${ }^{2}$ Department of Pathology, Xiangya Hospital, Central South University, Changsha, China; ${ }^{3}$ Department of Internal Medicine, Qidong County Hospital of Traditional Chinese Medicine, Hengyang, China; ${ }^{4}$ Department of Chest Radiotherapy, Hunan Cancer Hospital, Changsha, China

${ }^{*}$ Correspondence: kuangweilu8807@163.com

Received January 21, 2019 / Accepted July 9, 2019

\begin{abstract}
Glioma is one of the most common and aggressive malignant primary brain tumors with high recurrence rate and mortality rate and heavily depends on the angiogenesis. LncRNA H19 has many diverse biological functions, including the regulation of cell proliferation, differentiation and metabolism. Here, we aimed to investigate the molecular mechanism of lncRNA H19 affecting angiogenesis in glioma, which could help to uncover potential target for glioma therapy. RT-qPCR analysis was performed to detect the expression of lncRNA H19 and miR-138 in HEB, U87, A172 and U373 cell lines. MTT assay was used to evaluate the cell viability. To evaluate the migration and invasion after lncRNA H19 knockdown, Transwell and wound healing assay were employed. After lncRNA H19 knockdown, protein expression of HIF-1a and VEGF was detected by western blot and tube formation was assessed. For the prediction and validation of the interaction between lncRNA H19 and miR-138, bioinformatics and luciferase assay were performed. We investigated the regulatory roles and downstream molecular mechanisms of lncRNA H19 in glioma by H19 knockdown, which inhibited the proliferation, migration and angiogenesis of glioma cells. Moreover, miR-138 acted as a target of H19 as detected by luciferase reporter assays. Meanwhile, HIF-1 1 a was also a target of miR-138 and miR-138 could also regulate the proliferation, migration and angiogenesis of glioma cells by targeting HIF- $1 \alpha$ and affecting the expression of VEGF in turn. Our findings identified an upregulated lncRNA H19 in glioma cells, which could promote proliferation, migration, invasion and angiogenesis via miR-138/HIF-1 $\alpha$ axis as a ceRNA. This study provided a new opportunity to advance our understanding in the potential mechanism of lncRNA in glioma angiogenesis.
\end{abstract}

Key words: glioma, IncRNA, H19, miR-138, HIF-1 $\alpha$, VEGF

Glioma is one of the most common and aggressive primary brain tumors [1]. Due to invasive growth and difficult complete tumor resection, its 5-year survival rate is invariably poor [2]. Even though advances in treatment have made some difference in glioma therapy, the recurrence rate and mortality rate were still high and the mean life expectancy of patients with glioma undergoing conventional treatment was less than 15 months [3]. Moreover, the molecular mechanisms underlying glioma formation still remain elusive. Thus, it is essential to identify critical molecules involved in glioma tumorigenicity and angiogenesis, which may provide breakthroughs for glioma treatment.

Angiogenesis is characterized by the formation of new blood vessels from the existing vessels [4], which plays a critical role in the malignancy, development and progression of tumors. Angiogenesis fulfills the nutrient and oxygen requirements of the tumors making it a necessary prerequisite for tumor progression and invasion [5]. Human gliomas are rich in blood vessels and their growth and proliferation are heavily dependent on angiogenesis [6]. Vascular endothelial growth factor (VEGF) is the most important factor for inducing endothelial cell proliferation and is directly related to angiogenesis in tumors [7]. VEGF plays a leading role in promoting angiogenesis [8]. At present, HIF- $1 \alpha$ is one of the most studied upstream molecules of VEGF that affects the expression of VEGF in tumors angiogenesis [9].

Hypoxia-inducible transcription factors (HIFs) have been reported to play a crucial role in physiological adaptation to varying oxygenation states, which act as essential controls for adaptive changes at the cellular, tissue and organismal levels in response to a range of challenges, including altered tissue oxygenation $[10,11]$. Among them, HIF-1 $1 \alpha$ has been 
the most extensively studied one and plays a pivotal role in driving angiogenesis. However, the molecular mechanism of HIF-1 $\alpha$ /VEGF axis in glioma regulation is still unclear.

Long non-coding RNAs (lncRNAs) are non-coding RNAs longer than 200 nucleotides with limited coding potential, which play pivotal roles in the regulation of cellular biological behaviors such as cell proliferation, differentiation, metastasis and drug resistance [12]. Recently, studies also have shown that lncRNAs play regulatory role in glioma genesis [13]. IncRNA H19 has many diverse biological functions, participating in the regulation of cell proliferation, differentiation and metabolism [14]. Increased expression of H19 was observed in some cancers, such as bladder and gastric cancer, while decreased expression in others, such as hepatocellular carcinoma (HCC), which suggested that H19 may play its role in cancer both as an oncogene and as a tumor suppressor [15]. A previous study showed that $c-M y c$ could induce the expression of $\mathrm{H} 19$ in glioblastoma, which suggested that H19 may play a crucial role in glioblastoma development [16]. However, the exact mechanism is still elusive.

The present study investigated the expression of lncRNA H19 and miR-138 in glioma cells, and their role in glioma angiogenesis. We confirmed that H19 was upregulated in glioma cells and may function as a ceRNA to regulate HIF- $1 a$ by sponging miR-138 thus promoting angiogenesis of glioma cells. These findings provided a new insight of the regulatory role of lncRNA in the development of glioma.

\section{Materials and methods}

Cell lines and cell culture. Human glioma cell lines U87, A172 and U373 and human normal glial cell line HEB were obtained from American Type Culture Collection (ATCC, USA). Cells were cultured in the Dulbecco's modified Eagle's medium (DMEM, Gibco, USA) supplemented with $10 \%$ fetal bovine serum (FBS, Gibco, USA), $100 \mathrm{U} / \mathrm{ml}$ penicillin (HyClone, USA), and $100 \mu \mathrm{g} / \mathrm{ml}$ streptomycin (HyClone, USA) at $37^{\circ} \mathrm{C}$ in the presence of $5 \% \mathrm{CO}_{2}$.

Cell transfection. Short-hairpin H19 (sh-H19) and pcDNA-H19 plasmids and their respective negative controls (NC) were synthesized and purchased from Ribobio Biotech Co. (Guangzhou, China). miR-138 mimics, miR-138 inhibitor and their respective negative controls (NC) were synthesized and purchased from GenePharma (Suzhou, China). Cells were transiently transfected using Lipofectamine 3000 (Invitrogen, USA) when the confluence reached $70-80 \%$. Then, cells were collected at $48 \mathrm{~h}$ after transfection.

RNA extraction and RT-qPCR. Total RNA of cells was isolated using Trizol $^{\circ}$ (Invitrogen, USA) according to the manufacturer's protocol. ImProm-II Reverse Transcription System (Promega, USA) was used to generate Firststrand cDNA. Real-time qPCR of the reverse transcription products was determined using Permix Ex Taq (Takara, Japan), analyzed through the 7500 Real-time PCR System (Applied Biosystems, USA) and normalized with GAPDH or
U6, respectively. The relative expression levels of RNAs were calculated using the comparative $2^{-\Delta \Delta C T}$ method. All experiments were performed at least three times.

Primers used for qRT-PCR are as follows:

HIF-1a: F 5'- GTGGTGGTTACTCAGCACTTT-3',

R 5'- ATCTCCGTCCCTCAACCTCT-3';

VEGF: F 5'- TGTCTAATGCCCTGGAGCCT-3',

R 5' - TTAACTCAAGCTGCCTCGCC-3';

H19: F 5'- GCACCTTGGACATCTGGAGT-3',

R 5'- TTCTTTCCAGCCCTAGCTCA-3';

MiR-138: F 5'-AGCTGGTGTTGTGAATCAGGCCG-3',

R 5'-GCGAGCACAGAATTAATACGAC-3';

GAPDH: F 5'-AGGTCGGTGTGAACGGATTTG-3',

R 5'-GGGGTCGTTGATGGCAACA-3';

U6snRNA: F 5'-CTCGCTTCGGCAGCACA-3',

R 5'-AACGCTTCACGAATTTGCGT-3'.

Western blot analysis. Cells cultured in 6-well plates were collected and lysed with RIPA lysis buffer (Beotime, Shanghai, China). Protein concentration was measured by BCA Protein Assay Reagent Kit (Shenggong, China). After BCA quantification, equal amounts of protein were separated by $10 \%$ SDS-PAGE, electrophoretically transferred to nitrocellulose membrane (Millipore, USA), and incubated with primary antibodies: HIF-1 $\alpha$ (Abcam, USA, 1:1000), VEGF (Abcam, USA, 1:1000), GAPDH (Abcam, USA, 1:3000). The membrane was washed with TBST containing $0.1 \%$ Tween 20 for three times. And then membrane was incubated with HRP-labeled goat anti-mouse/rabbit IgG (1:5000, Sigma) for $2 \mathrm{~h}$ at room temperature. Signals were visualized with SuperPico chemiluminescent substrate (Pierce, USA) and film exposure.

Luciferase reporter assay. Starbase software, was used to predict the interaction probability and the binding site of H19, miR-138 and HIF-1a. Then binding site of H19 and the 3' UTR fragment of HIF- $1 \alpha$ as well as their mutant sequences were subcloned into the pGL3-control vector (Promega, USA) immediately downstream of the stop codon of the luciferase gene. After cells were seeded into 24-well plates and grown overnight to $80-90 \%$ confluence, plasmids were transiently co-transfected with $5 \mathrm{ng}$ miR-138 mimics or mimic NC into cells using Lipofectamine 3000 (Invitrogen, USA) according to the manufacturer's manual. $48 \mathrm{~h}$ after transfection, the luciferase activity was assessed using the Dual-Luciferase Reporter Assay Kit (Promega, USA).

Tube formation assay. Tube formation assay was conducted as described before. Briefly, the 96-well plates were coated with $100 \mu \mathrm{l}$ Matrigel (BD Biosciences, USA) per well and maintained at $37^{\circ} \mathrm{C}$ for $30 \mathrm{~min}$. After that, U87 cells and HUVEC cells were then added to wells which were re-suspended in $100 \mu \mathrm{l}$ complete DMEM medium at the concentration of $4 \times 10^{5} / \mathrm{ml}$ and incubated at $37^{\circ} \mathrm{C}$ for $24 \mathrm{~h}$. Fluorescence microscopy (DP71, Olympus, Japan) was applied to collect the photos with Chemi Imager 5500 V2.03 software (Alpha Innotech, USA) used to measure the numbers of tubule branches. 
In vitro matrigel invasion assay. In vitro matrigel invasion assay was conducted as described before [17]. Briefly, the upper chamber of a 24-well transwell chamber ( $8 \mu \mathrm{m}$ pore size, Corning, USA) was used to incubate U87 cells re-suspended in $200 \mu \mathrm{l}$ serum-free medium at a density of $4 \times 10^{5}$ cells $/ \mathrm{ml}$. Then, $600 \mu \mathrm{l}$ of the medium containing $5 \%$ FBS and U87 cells was added to the lower chamber. $48 \mathrm{~h}$ after incubation at $37^{\circ} \mathrm{C}$, the cells on the upper membrane surface were removed. Cells on the lower surface of the membrane were then fixed with methanol and glacial acetic acid at the ratio of 3:1 and stained with 10\% Giemsa (Dinguo, China). Five randomly selected fields were counted for statistical analysis in each well.

Wound-healing assay. Wound-healing assay was conducted as described before [18]. Briefly, cells were grown to $90 \%$ confluence in a 6 -well plate at $37^{\circ} \mathrm{C}$ in the presence of $5 \% \mathrm{CO}_{2}$. Then, cells were scratched with sterile $10 \mu \mathrm{l}$ pipette tip to create a wound. Cells were then washed twice with PBS to remove floating cells and added to a medium without serum. Photos of the wound were taken with $\times 100$ magnification.

Statistical analysis. Statistical analysis was performed by GraphPad Prism 5. Group distributions were conducted with the Student's t test or one-way ANOVA analysis and a paired t-test was performed to compare continuous variables between paired control and experiment group. Value of $\mathrm{p}<0.05$ was considered statistically significant. All experiments in this study were in triplicated.

\section{Results}

HIF-1 $\alpha$ and VEGF are significantly up-regulated in glioma cells. First, we investigated the expression of HIF-1 $\alpha$ and VEGF, two angiogenic factors on the mRNA level in three glioma cell lines U87, A172 and U373 as well as the human normal glial cell line HEB by qPCR. As shown in Figure 1A, both HIF-1 $\alpha$ and VEGF were significantly overexpressed in glioma cell lines compared with HEB cells. Then, the protein levels of HIF-1 $\alpha$ and VEGF were also tested in those cell lines by WB. As shown in Figure 1B, HIF-1 $\alpha$ and VEGF proteins were also upregulated in those glioma cells. Taken together, these results indicated that differentially expressed HIF- $\alpha$ / VEGF axis in glioma cells was a potential therapeutic target.

lncRNA H19 promotes angiogenesis of glioma. To explore the relationship between abnormal expression of HIF-1a/VEGF and lncRNA H19, we then investigated the expression of lncRNA H19 in three glioma cell lines U87, A172 and U373 as well as the human normal glial cell line HEB by qPCR. As shown in Figure 2A, H19 was significantly overexpressed in glioma cell lines compared with HEB cells. After H19 knockdown or H19 overexpression, the results of tube formation assay suggested that number of branches was decreased in sh-H19 group compared with NC group while significantly increased in H19 overexpression group, which indicated that $\mathrm{H} 19$ significantly promoted tube formation of glioma cells (Figure 2B). Cell proliferation of U87 cells was also tested by MTT assay, which showed that the cell viability was decreased in sh-H19 group compared with sh-NC group and the cell viability was increased in pcDNA-H19 group. Furthermore, the expression of HIF-1 $\alpha$ and VEGF was also tested in sh-H19 and pcDNA-H19 groups by qPCR and WB. Both HIF-1 $\alpha$ and VEGF were decreased in sh-H19 group compared with NC group while they were significantly increased in H19 overexpression group on the mRNA level (Figure 2D) and protein level (Figure 2E). In general, overexpression of lncRNA H19 in gliomas may result in increased expression of VEGF, leading to increased angiogenesis.

lncRNA H19 directly interacts with miR-138. To explore the mechanism by which lncRNA H19 regulates angiogenesis in gliomas, we investigated the expression of miR-138 in three glioma cell lines U87, A172 and U373 as well as the human normal glial cell line HEB by qPCR. As shown in Figure 3A, miR-138 was significantly decreased in glioma cell lines compared with HEB cells. Furthermore, the expression of miR-138 was also tested in sh-H19 and pcDNA-H19 groups by qPCR (Figure 3B), which showed that miR-138 was upregulated in sh-H19 group and downregulated in lncRNA H19 overexpression group. We predicted the potential lncRNA H19 binding region on miR-138 by bioinformatics. As shown in Figure 3C, that miR-138 was predicted to directly bind with lncRNA H19. To determine whether H19 directly regulates miR-138, luciferase reporters of H19
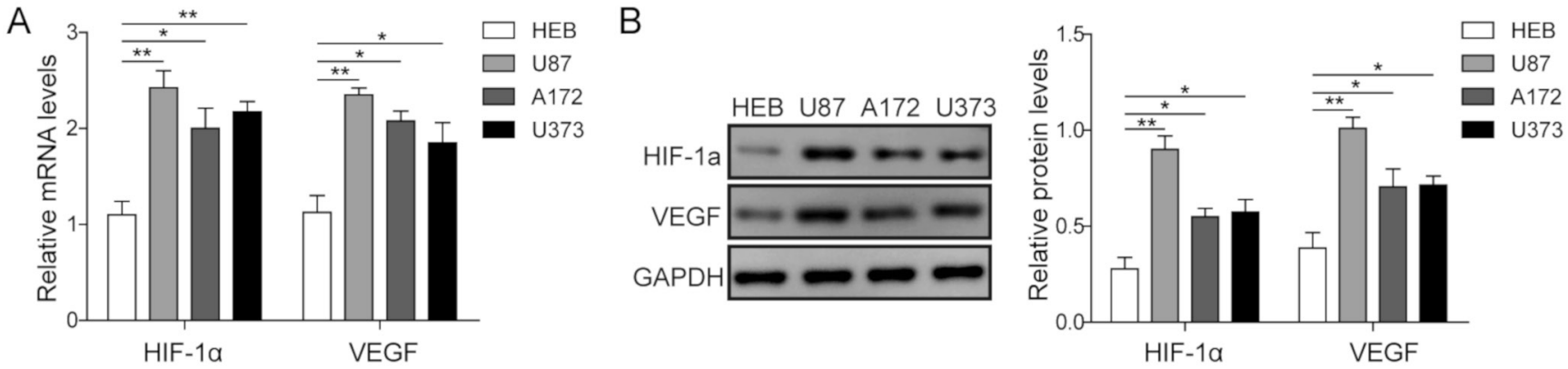

Figure 1. The expression of HIF-1 $\alpha$ and VEGF in glioma cells. A) The expression level of HIF-1 $\alpha$ and VEGF in glioma cells by qPCR. GAPDH was used for normalization. B) The expression level of HIF- $1 \alpha$ and VEGF in glioma cells by WB. ${ }^{*} \mathrm{p}<0.05,{ }^{* *} \mathrm{p}<0.01$. 


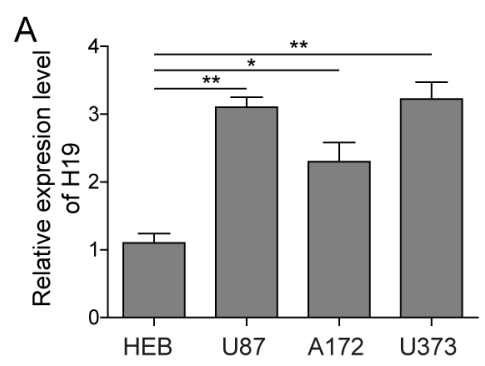

B
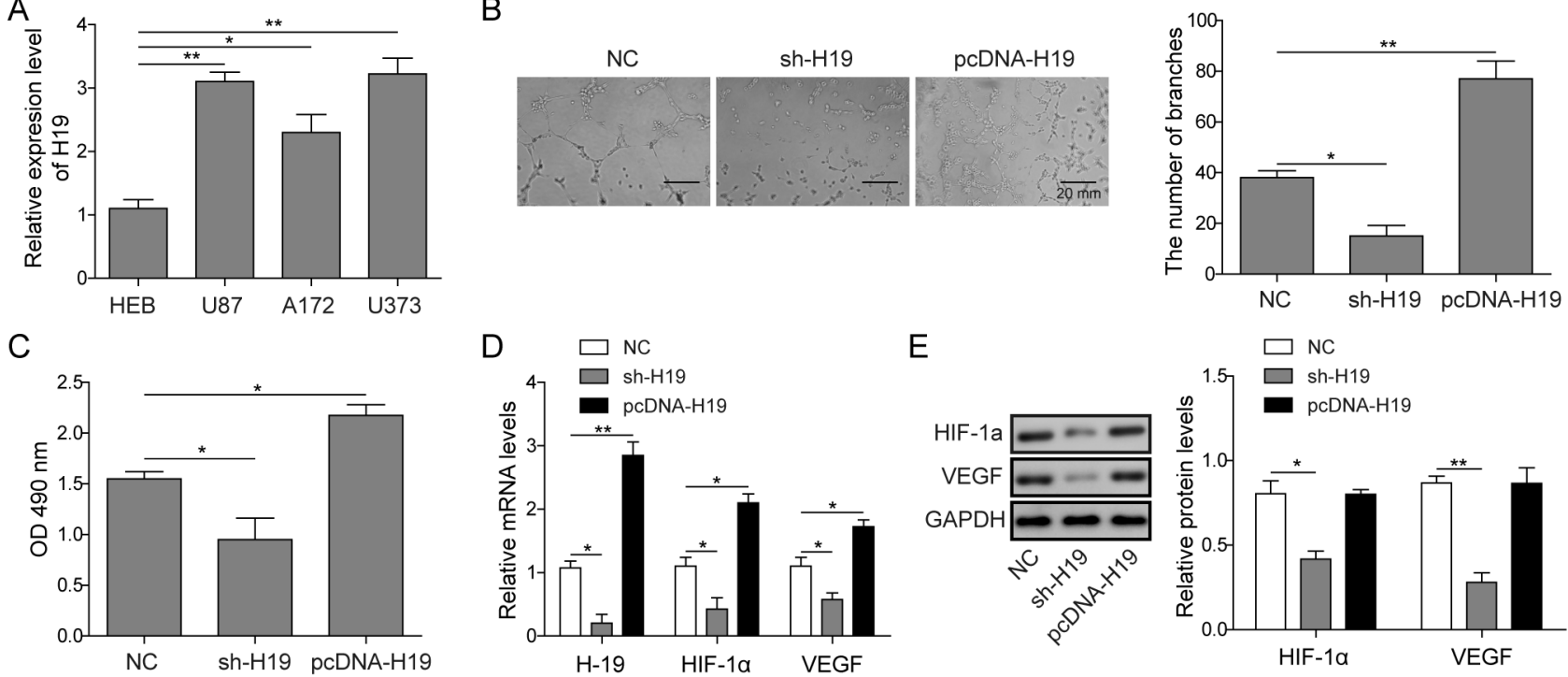

Figure 2. Regulation of IncRNA H19 on angiogenesis. A) The expression level of IncRNA H19 in glioma cells by qPCR. GAPDH was used for normalization. B) Effect of IncRNA H19 of U87 cells on tube formation. Data are presented as mean \pm SD. (n=5, each group). C) Effect of 1 ncRNA H19 on U87 cell proliferation. Data are presented as mean \pm SD. ( $n=5$, each group). D) The expression level of H19, HIF-1 $\alpha$ and VEGF in U87 cells after H19 knockdown and overexpression by qPCR. GAPDH was used for normalization. E) The expression level of HIF-1 $\alpha$ and VEGF in glioma cells after H19 knockdown and overexpression by WB. GAPDH was used for normalization. ${ }^{*} \mathrm{p}<0.05,{ }^{* *} \mathrm{p}<0.01$.

A

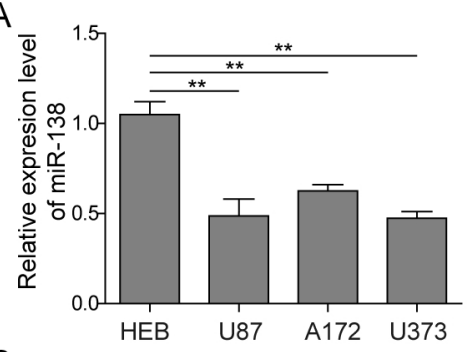

B

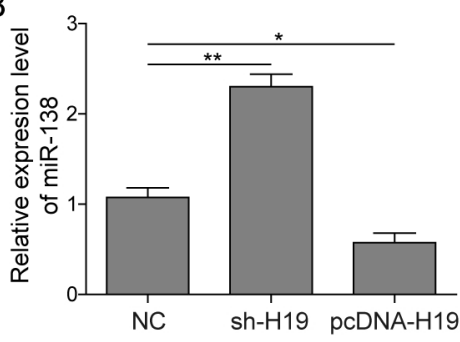

C

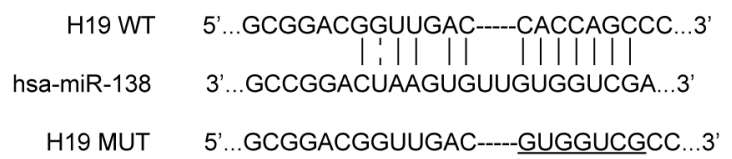

D

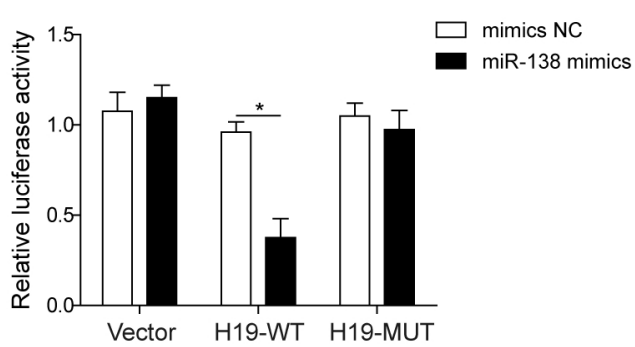

Figure 3. miR-138 is the target of $\mathrm{H} 19$ in glioma cells. A) The expression level of miR-138 in glioma cells by qPCR. U6 was used for normalization. B) The expression level of miR-138 in U87 cells after H19 knockdown and overexpression by qPCR. U6 was used for normalization. C) The predicted binding site between the IncRNA H19 and miR-138 by the Starbase software, and the bold part is the mutated base sequence. Luciferase reporter constructs of H19 binding site (H19-WT) and a mutated form (H19-MUT). D) The luciferase activity of the H19-WT and H19-MUT in HEK-293T cells treated with miR-138 mimics or NC. ${ }^{*} \mathrm{p}<0.05,{ }^{* *} \mathrm{p}<0.01$.

RNA (H19-WT), and a mutated form (H19-MUT) were constructed (Figure 3C). We demonstrated that miR-138 mimics significantly suppressed the luciferase activity of the H19-WT in HEK-293T cells with no effect observed on the mutated form (Figure 3D), which indicated a direct interaction of $\mathrm{H} 19$ with miR-138 in this putative binding site. Taken together, miR-138 is abnormally expressed in glioma and has a direct negative regulatory relationship with lncRNA H19, suggesting that miR-138 may be involved in lncRNA H19 regulation of glioma angiogenesis.

miR-138 inhibits angiogenesis via targeting HIF-1 $\alpha /$ VEGF axis. To further explore the role of miR-138 in the regulation of angiogenesis by lncRNA H19, we investigated the relationship between miR-138 and HIF-1a/VEGF axis. 
MiR-138 mimics and miR-138 inhibitor were transfected to U87 cells. Figure 4A indicated that HIF-1a was significantly decreased by miR-138 mimics while it was significantly upregulated by miR-138 inhibitor both on mRNA level and protein level. Bioinformatics analysis found the potential binding site between miR-138 and HIF-1a (Figure 4B). Dual luciferase reporter assay was further carried out. When treated with miR-138 mimics, the relative luciferase activity was significantly decreased in HIF-1 $\alpha$-WT but didn't change in the HIF-1 $a$-MUT (Figure 4C). These results suggested that miR-138 could directly and negatively regulate the expression of HIF- $1 \alpha$ through binding the 3' UTR region of HIF-1 1 . To test whether miR-138 could regulate angiogenesis through targeting HIF- $1 \alpha$, tube formation assay was carried out after miR-138 mimics or inhibitor was transfected in U87 cells. Figure $4 \mathrm{D}$ showed that miR-138 mimics decreased tubule formation and relative number of branches while HIF-1a overexpression compensated this effect in U87 cells. On the other hand, miR-138 inhibitor increased tubule formation and relative number of branches while HIF-1a knockdown compensated this effect in U87 cells. Taken together, these results indicated that miR-138 was downregulated in gliomas and regulated angiogenesis in gliomas by targeting HIF-1a/ VEGF axis.
H19 regulates angiogenesis through miR-138/HIF-1a axis. Next, we test whether H19 regulates angiogenesis of glioma cells through miR-138/HIF-1 $\alpha$ axis. Figure 5A showed that $\mathrm{H} 19$ overexpression could increase the expression of HIF- $1 \alpha$ and VEGF while miR-138 mimics could rescue this effect in U87 cells. On the other hand, H19 knockdown could decreased the expression of HIF- $1 \alpha$ and VEGF while miR-138 inhibitor rescued this effect in U87 cells. Tube formation assay was also carried out in HUVEC cells, and Figure 5B showed that miR-138 inhibitor, HIF-1 $\alpha$ or VEGF compensated the negative effect on number of branches by H19 knockdown. Furthermore, the results of transwell invasion assay showed that miR-138 inhibitor, HIF-1a or VEGF could rescue the positive effect of cell invasion by $\mathrm{H} 19$ knockdown in U87 cells (Figure 5C). Correspondingly, wound-healing assay also showed that miR-138 inhibitor, HIF-1a or VEGF could rescue the positive effect on migration of U87 cells by H19 knockdown (Figure 5D). Taken together, these results demonstrated that overexpression of IncRNA H19 in gliomas could promote glioma angiogenesis by downregulating the expression of miR-138, thus upregulating HIF-1a/VEGF expression. While knockdown of lncRNA H19 or transfection of miR-138 mimics can inhibit angiogenesis caused by lncRNA H19.

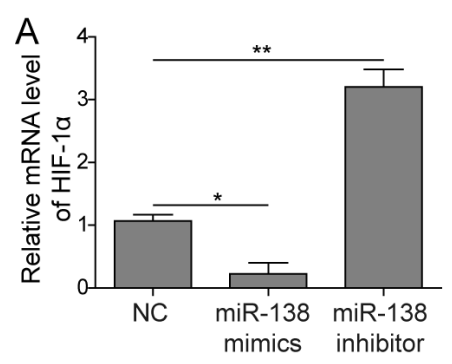

C
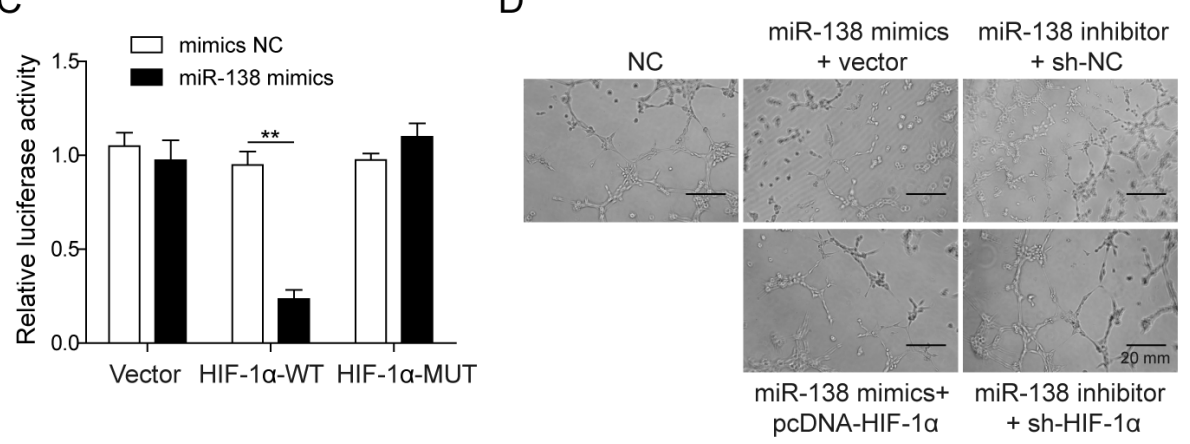

B
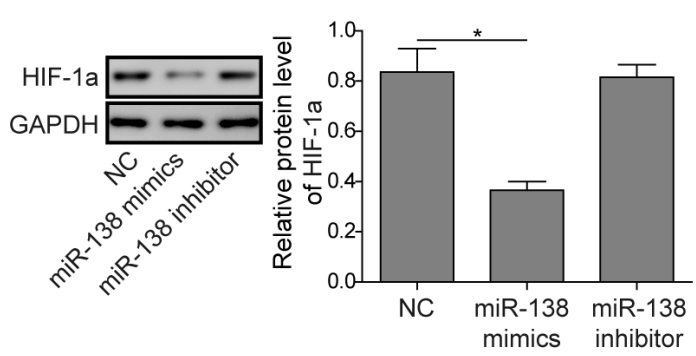

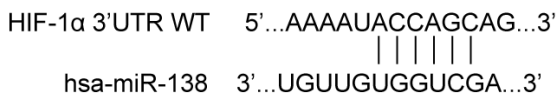

HIF-1a 3'UTR WT 5'...AAAAUACCAGCAG...3 || |||

hsa-miR-138 3'...UGUUGUGGUCGA...3'

HIF-1a 3'UTR MUT 5'...AAAAUUGGUCGAG...3'

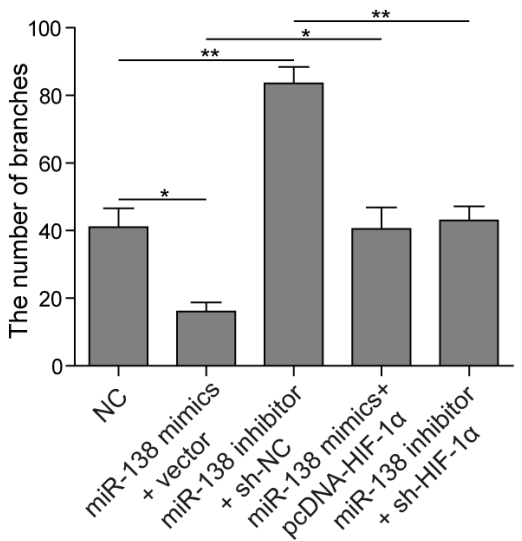

Figure 4. miR-138 regulates angiogenesis through target HIF-1 $\alpha$. A) The expression level of HIF-1 $\alpha$ in U87 cells transfected with miR-138 mimics and inhibitor by QPCR and WB. GAPDH was used for normalization. B) The predicted binding site between HIF-1a and miR-138 by Targetscan software, and the bold part is the mutated base sequence. Luciferase reporter constructs of HIF-1a binding site (HIF-1 $\alpha$-WT) and a mutated form (HIF-1aMUT). C) The luciferase activity of the HIF-1 $\alpha$-WT and HIF-1 $\alpha$-MUT in HEK-293T cells treated with miR-138 mimics or NC. D) Tube formation assay after co-transfected miR-138 mimics with pcDNA-HIF-1 $\alpha$ and empty vector as well as miR-138 inhibitor with sh-HIF-1 $\alpha$ and NC. Data are presented as mean \pm SD. $\left(n=5\right.$, each group). ${ }^{*} \mathbf{p}<0.05,{ }^{* *} \mathrm{p}<0.01$. 

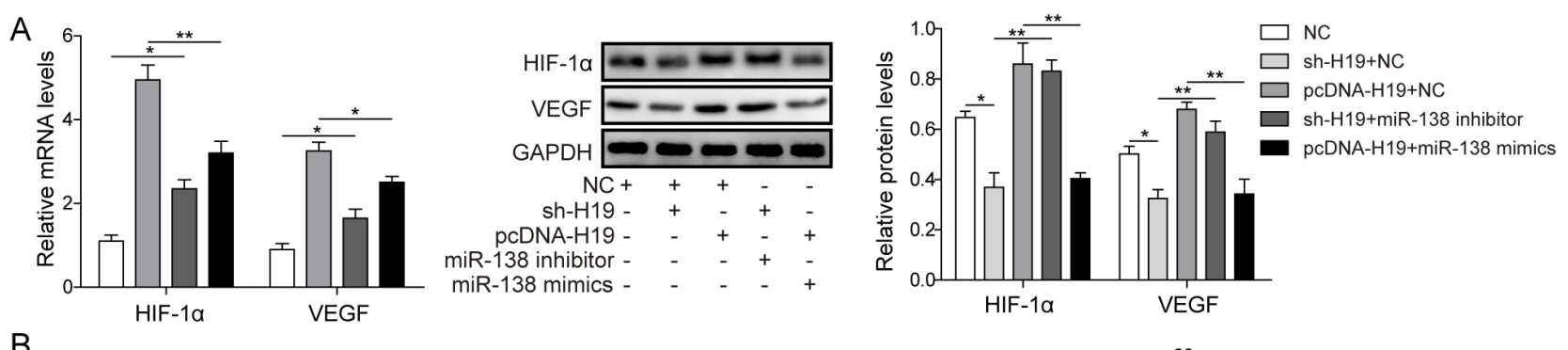

$\mathrm{B}$
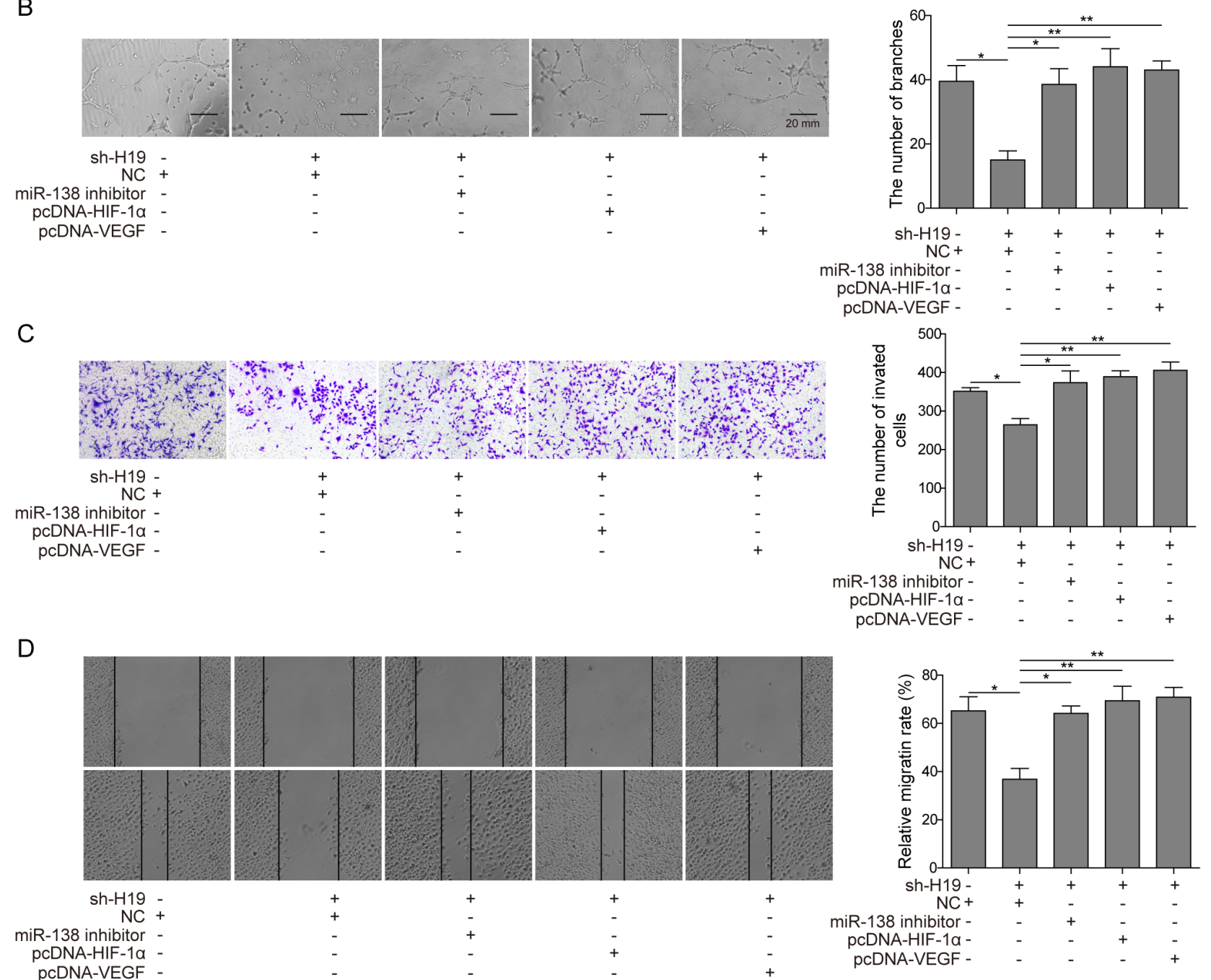

Figure 5. IncRNA H19 regulates angiogenesis through miR-138/HIF-1a/VEGF axis. A) The expression of HIF-1a and VEGF in U87 cells and U87 cells transfected with miR-138 inhibitor after H19 knockdown as well as transfected with miR-138 mimics after H19 overexpression by qPCR and WB. GAPDH was used for normalization. B) Tube formation assay of HUVEC cells and U87 cells transfected with miR-138 inhibitor, pcDNA-HIF-1 $\alpha$ or pcDNA-VEGF after H19 knockdown. Data are presented as mean \pm SD. (n=5, each group). C) The effect of H19 knockdown on cell invasion of U87 cells and U87 cells transfected with miR-138 inhibitor, pcDNA-HIF-1 $\alpha$ or pcDNA-VEGF was assessed by a transwell assay. D) The effect of H19 knockdown on cell migration of U87 cells and U87 cells transfected with miR-138 inhibitor, pcDNA-HIF-1 $\alpha$ or pcDNA-VEGF was assessed by a wound-healing assay. ${ }^{\star} \mathbf{p}<0.05,{ }^{* *} \mathrm{p}<0.01$.

\section{Discussion}

Angiogenesis is required for tumor progression and is a balanced process controlled by pro- and anti-angiogenic molecules ${ }^{5}$. VEGFA is a well-known pro-angiogenic factor and is upregulated in gliomas [14]. In tumors, vessel abnormalities included the increased permeability and hemorrhage, leading to tumor hypoxia and increased metastasis [19]. Therefore, angiogenic factors related to VEGF signaling were also required for functional angiogenesis and preven- 
tion of excessive angiogenesis [20]. Hence, productive angiogenesis relied on balanced release of angiogenic and angiostatin factors from malignant and stromal cell types [20]. Accumulating evidence showed that VEGFA was upregulated in gliomas through multiple mechanisms $[6,21,22]$. Low oxygen concentration in growing gliomas upregulated the expression of HIF-1 $\alpha$, which increased VEGFA mRNA level [6]. While, the brain-derived neurotrophin factor and integrin linked kinase 1 (ILK1) increased VEGFA expression by stimulating HIF-1 [9]. Lately, the anti-angiogenic therapy, a brand new therapeutic strategy, had presented a deep involvement in treatment of cancers including glioma [23]. However, most of the anti-angiogenic drugs were currently target VEGF, and the cells were prone to drug resistance [24]. Thus, this article aimed to explore the molecular mechanism of glioma angiogenesis.

LncRNA H19 was reported to participate in cancer both as an oncogene and as a tumor suppressor [15]. Downregulation of H19 was reported to contribute to the proliferation and migration of papillary thyroid carcinoma [25]. H19 was also reported to be a major mediator of doxorubicin chemoresistance in breast cancer cells [26]. Previous study also showed that $\mathrm{H} 19$ might play important roles in angiogenesis. H19 could upregulate VEGFA to enhance angiogenic capacity of mesenchymal stem cells [27]. Recently, reports showed that increased level of H19 could promote invasion and angiogenesis of glioblastoma cells [28]. Consistent with those studies, we also uncovered that lncRNA H19 was overexpressed in gliomas and affected angiogenesis by upregulating the expression of HIF-1a/VEGF.

Recently, some lncRNAs containing miRNA-binding sites could act as miRNA sponges. In previous study, H19 was reported to mediate EMT by sponging miR-130a-3p in glioma [29]. miR-138 expresses aberrantly in different cancers and acts as a tumor suppressor by targeting many target genes related to proliferation, apoptosis, invasion and migration [29-31]. In this study, we found that miR-138 was downregulated in glioma cells and the expression of miR-138 was upregulated after $\mathrm{H} 19$ knockdown. We further validated that miR-138 could directly bind with H19 and HIF-1a through bioinformatics analysis and luciferase assays. Taken together, these data indicated that lncRNA H19 modulated miR-138 level through its function as a miRNA sponge to trap miR-138, therefore regulated the expression of HIF-1a and promoted angiogenesis in glioma (Figure 6).

The de novo generation of new blood vessels was one of the most prominent features of glioma and the progression of glioma depends on the nourishment of blood vessels [17, 32]. However, there were still few studies on angiogenesis in glioma. Thus, it might provide breakthroughs for glioma treatment to identify of critical molecules involved in glioma tumorigenicity and angiogenesis. Although we initially revealed a novel downstream molecular mechanism of angiogenesis in glioma by in vitro experiments, there is still much to be done to deeply understand this complex mechanism. As

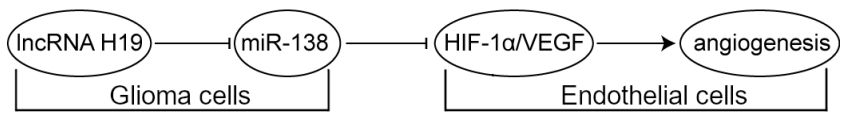

Figure 6. IncRNA H19 promotes glioma angiogenesis through miR-138/ HIF-1a/VEGF axis. In glioma cells, IncRNA H19 modulated miR-138 level through its function as a miRNA sponge to trap miR-138, therefore upregulated the expression of HIF-1 $\alpha$ and promoted angiogenesis in glioma.

the situation in the animal body is more complicated and has more uncertainty than cell models, whether this mechanism could be applied to an animal model is not certain. Therefore, in the future, this novel molecular mechanism needs further verification in mice model prove its potential of therapeutic targets, or even be correlated with patient prognosis through samples of cancer patients.

Taken together, we uncovered that expression of lncRNA H19 was increased and miR-138 expression was decreased in glioma cells. Besides, downregulation of H19 suppressed cell proliferation, invasion and angiogenesis by sponging miR-138. This study provided important clues to understand the molecular mechanism of lncRNA H19 in regulation of angiogenesis in glioma, which illuminated a potential therapeutic target for glioma in the future.

Acknowledgements: This work was supported by National Natural Science Foundation of China (NO. 81803582).

\section{References}

[1] ANGELOPOUlOU E, PIPERI C. Emerging role of plexins signaling in glioma progression and therapy. Cancer Lett 2018; 414: 81-87. https://doi.org/10.1016/j.canlet.2017.11.010

[2] YAMAGUCHI F. Therapy Decisions for Patients with HighGrade Glioma and Their Families. World Neurosurg 2017; 102: 671-672. https://doi.org/10.1016/j.wneu.2017.03.094

[3] DAVIS ME. Glioblastoma: Overview of Disease and Treatment. Clin J Oncol Nurs 2016; 20: S2-8. https://doi. org/10.1188/16.CJON.S1.2-8

[4] BETZ C, LENARD A, BELTING HG, AFFOLTER M. Cell behaviors and dynamics during angiogenesis. Development 2016; 143: 2249-2260. https://doi.org/10.1242/dev.135616

[5] NISHIDA N, YANO H, NISHIDA T, KAMURA T, KOJIRO M. Angiogenesis in cancer. Vasc Health Risk Manag 2006; 2: 213-219. https://doi.org/10.2147/vhrm.2006.2.3.213

[6] MA X, LI Z, LI T, ZHU L, LI Z et al. Long non-coding RNA HOTAIR enhances angiogenesis by induction of VEGFA expression in glioma cells and transmission to endothelial cells via glioma cell derived-extracellular vesicles. Am J Transl Res 2017; 9: 5012-5021. 
[7] VERHEUL HM, PINEDO HM. The role of vascular endothelial growth factor (VEGF) in tumor angiogenesis and early clinical development of VEGF-receptor kinase inhibitors. Clin Breast Cancer 2000; 1 Suppl 1: S80-84. https://doi. org/10.3816/cbc.2000.s.015

[8] SHIBUYA M. Vascular Endothelial Growth Factor (VEGF) and Its Receptor (VEGFR) Signaling in Angiogenesis: A Crucial Target for Anti- and Pro-Angiogenic Therapies. Genes Cancer 2011; 2: 1097-1105. https://doi. org/10.1177/1947601911423031

[9] LIN C, MCGOUGH R, ASWAD B, BLOCK JA, TEREK R. Hypoxia induces HIF-1alpha and VEGF expression in chondrosarcoma cells and chondrocytes. J Orthop Res 2004; 22: 1175-1181. https://doi.org/10.1016/j.orthres.2004.03.002

[10] PALAZON A, TYRAKIS PA, MACIAS D, VELIÇA P, RUNDQVIST $\mathrm{H}$ et al. An HIF-1alpha/VEGF-A Axis in Cytotoxic T Cells Regulates Tumor Progression. Cancer Cell 2017; 32: 669-683.e5. https://doi.org/10.1016/j. ccell.2017.10.003

[11] SEMENZA GL. Hypoxia-inducible factors: mediators of cancer progression and targets for cancer therapy. Trends Pharmacol Sci 2012; 33: 207-214. https://doi.org/10.1016/j. tips.2012.01.005

[12] SHEN Y, LIU S, FAN J, JIN Y, TIAN B et al. Nuclear retention of the lncRNA SNHG1 by doxorubicin attenuates hnRNPC-p53 protein interactions. EMBO Rep 2017; 18: 536-548. https://doi.org/10.15252/embr.201643139

[13] CAI H, LIU X, ZHENG J, XUE Y, MA J et al. Long noncoding RNA taurine upregulated 1 enhances tumor-induced angiogenesis through inhibiting microRNA-299 in human glioblastoma. Oncogene 2017; 36: 318-331. https://doi. org/10.1038/onc.2016.212

[14] LIU FT, PAN H, XIA GF, QIU C, ZHU ZM. Prognostic and clinicopathological significance of long noncoding RNA H19 overexpression in human solid tumors: evidence from a meta-analysis. Oncotarget 2016; 7: 83177-83186. https://doi. org/10.18632/oncotarget.13076

[15] RAVEH E, MATOUK IJ, GILON M, HOCHBERG A. The H19 Long non-coding RNA in cancer initiation, progression and metastasis - a proposed unifying theory. Mol Cancer 2015; 14: 184. https://doi.org/10.1186/s12943-015-0458-2

[16] BARSYTE-LOVEJOY D, LAU SK, BOUTROS PC, KHOSRAVI F, JURISICA I et al. The c-Myc oncogene directly induces the H19 noncoding RNA by allele-specific binding to potentiate tumorigenesis. Cancer Res 2006; 66: 5330-5337. https://doi.org/10.1158/0008-5472.CAN-06-0037

[17] YANG C, ZHENG J, XUE Y, YU H, LIU X et al. The Effect of MCM3AP-AS1/miR-211/KLF5/AGGF1 Axis Regulating Glioblastoma Angiogenesis. Front Mol Neurosci 2018; 10: 437. https://doi.org/10.3389/fnmol.2017.00437

[18] PAN MH, CHIOU YS, CHEN WJ, WANG JM, BADMAEV $\mathrm{V}$ et al. Pterostilbene inhibited tumor invasion via suppressing multiple signal transduction pathways in human hepatocellular carcinoma cells. Carcinogenesis 2009; 30: 1234 1242. https://doi.org/10.1093/carcin/bgp121

[19] ELTZSCHIG HK, CARMELIET P. Hypoxia and inflammation. N Engl J Med 2011; 364: 656-665. https://doi. org/10.1056/NEJMra0910283
[20] NIU G, CHEN X. Vascular endothelial growth factor as an anti-angiogenic target for cancer therapy. Curr Drug Targets 2010; 11: 1000-1017. https://doi. org/10.2174/138945010791591395

[21] HU Y, LI Y, WU C, ZHOU L, HAN X et al. MicroRNA-140-5p inhibits cell proliferation and invasion by regulating VEGFA/MMP2 signaling in glioma. Tumour Biol 2017; 39: 1010428317697558. https://doi. org/10.1177/1010428317697558

[22] LIU Q, LIAO F, WU H, CAI T, YANG L et al. Different expression of miR-29b and VEGFA in glioma. Artif Cells Nanomed Biotechnol 2016; 44: 1927-1932. https://doi.org/ $10.3109 / 21691401.2015 .1111237$

[23] WICK W, PLATTEN M, WICK A, HERTENSTEIN A, RADBRUCH A et al. Current status and future directions of anti-angiogenic therapy for gliomas. Neuro Oncol 2016; 18: 315-328. https://doi.org/10.1093/neuonc/nov180

[24] VASUDEV N, REYNOLDS AR. Anti-angiogenic therapy for cancer: current progress, unresolved questions and future directions. Angiogenesis 2014; 17: 471-494. https://doi. org/10.1007/s10456-014-9420-y

[25] LAN X, SUN W, DONG W, WANG Z, ZHANG T et al. Downregulation of long noncoding RNA H19 contributes to the proliferation and migration of papillary thyroid carcinoma. Gene 2018; 646: 98-105. https://doi.org/10.1016/j. gene.2017.12.051

[26] ZHU QN, WANG G, GUO Y, PENG Y, ZHANG R et al. LncRNA H19 is a major mediator of doxorubicin chemoresistance in breast cancer cells through a cullin4A-MDR1 pathway. Oncotarget 2017; 8: 91990-92003. https://doi. org/10.18632/oncotarget.21121

[27] HOU J, WANG L, WU Q, ZHENG G, LONG H et al. Long noncoding RNA H19 upregulates vascular endothelial growth factor A to enhance mesenchymal stem cells survival and angiogenic capacity by inhibiting miR-199a-5p. Stem Cell Res Ther 2018; 9: 109. https://doi.org/10.1186/s13287018-0861-X

[28] JIANG X, YAN Y, HU M, CHEN X, WANG Y et al. Increased level of H19 long noncoding RNA promotes invasion, angiogenesis, and stemness of glioblastoma cells. J Neurosurg 2016; 2016: 129-136. https://doi.org/10.3171/2014.12. JNS1426.test

[29] QU M, ZHU Y, JIN M. MicroRNA-138 inhibits SOX12 expression and the proliferation, invasion and migration of ovarian cancer cells. Exp Ther Med 2018; 16: 1629-1638. https://doi.org/10.3892/etm.2018.6375

[30] SHA HH, WANG DD, CHEN D, LIU SW, WANG Z et al. MiR-138: A promising therapeutic target for cancer. Tumour Biol 2017; 39: 1010428317697575. https://doi. org/10.1177/1010428317697575

[31] ZHOU Z, LI Z, SHEN Y, CHEN T. MicroRNA-138 directly targets TNFAIP8 and acts as a tumor suppressor in osteosarcoma. Exp Ther Med 2017; 14: 3665-3673. https://doi. org/10.3892/etm.2017.4947

[32] JOVCEVSKA I, KOCEVAR N, KOMEL R. Glioma and glioblastoma - how much do we (not) know? Mol Clin Oncol 2013; 1: 935-941. https://doi.org/10.3892/mco.2013.172 